\title{
An alternative menaquinone biosynthetic pathway operating in microorganisms: an attractive target for drug discovery to pathogenic Helicobacter and Chlamydia strains
}

\author{
Tohru Dairi
}

Menaquinone is an essential vitamin as an obligatory component of the electron transfer pathway in microorganisms. Menaquinone has been shown to be derived from chorismate by eight enzymes, designated MenA to $-\mathrm{H}$ in Escherichia coli. However, bioinformatic analyses of whole-genome sequences have suggested that some microorganisms, such as Helicobacter pylori and Campylobacter jejuni, which are known to cause gastric carcinoma and diarrhea, respectively, do not have orthologs of most of the men genes, although they synthesize menaquinone. The ${ }^{13} \mathrm{C}$-labeling pattern of menaquinone purified from Streptomyces coelicolor $\mathrm{A} 3(2)$ grown on $\left[\mathrm{U}^{13} \mathrm{C}\right]$ glucose was quite different from that of $E$. coli, suggesting that an alternative pathway was operating in the strain. We searched for candidate genes participating in the alternative pathway by in silico screening, and the involvement of these genes in the pathway was confirmed by gene-disruption experiments. We also used mutagenesis to isolate mutants that required menaquinone for their growth and used these mutants as hosts for shotgun cloning experiments. Metabolites that accumulated in the culture broth of mutants were isolated and their structures were determined. Taking these results together, we deduced the outline of the alternative pathway, which branched at chorismate in a similar manner to the known pathway but then followed a completely different pathway. As humans and some useful intestinal bacteria, such as lactobacilli, lack the alternative pathway, it would be an attractive target for the development of chemotherapeutics.

The Journal of Antibiotics (2009) 62, 347-352; doi:10.1038/ja.2009.46; published online 26 June 2009

Keywords: biosynthesis; futalosine pathway; menaquinone; vitamin $\mathrm{K}$

\section{INTRODUCTION}

In prokaryotes, ubiquinone and menaquinone (MK; vitamin $\mathrm{K}$ ) are lipid-soluble molecules that shuttle electrons between membranebound protein complexes in the electron transport chain., ${ }^{1,2}$ For example, Escherichia coli, a facultative anaerobe, utilizes ubiquinone (CoQ-8) under aerobic conditions, but uses MK (MK-8) when grown anaerobically. ${ }^{2}$ On the other hand, Bacillus subtilis, a Gram-positive aerobe, only contains MK (MK-7). Therefore, MK biosynthesis is essential for the survival of B. subtilis. ${ }^{1,2}$ In mammalian cells, ubiquinone has a single role in the electron transport chain, which is located in the inner mitochondrial membrane. ${ }^{2}$ On the other hand, MK functions as an essential vitamin for the biological activation of a family of proteins involved in blood coagulation, ${ }^{3}$ bone metabolism, ${ }^{4}$ tissue calcification and cell cycle regulation. ${ }^{5}$

The biosynthesis of MK has mainly been studied in E. coli. ${ }^{1,2}$ Chorismate, derived from the shikimate pathway, is initially converted into isochorismate by MenF, isochorismate synthase, and then into 2-succinyl-6-hydroxy-2,4-cyclohexadiene-1-carboxylate by MenD, a thiamin-dependent enzyme. The latter compound is dehydrated by
MenC to yield an aromatic compound, o-succinylbenzoate (OSB), followed by the attachment of coenzyme A by MenE to yield $o$-succinylbenzoyl-CoA. $o$-Succinylbenzoyl-CoA is then converted into 1,4-dihydroxy 2-naphthoic acid by MenB. In the last two steps of the pathway, MK is synthesized by MenA and MenG, which catalyze a prenylation and an $S$-adenosylmethionine-dependent methylation, respectively (Figure 1A).

Approximately 6 years ago, however, I found that some bacteria do not possess orthologs of the men $\mathrm{B},-\mathrm{C},-\mathrm{D},-\mathrm{E}$ and $-\mathrm{F}$ genes by bioinformatic analyses and started to investigate an alternative pathway. On the basis of bioinformatic screenings, gene knockouts, shotgun cloning with isolated mutants and in vitro studies with recombinant enzymes, we have deduced the outline of the alternative pathway. ${ }^{6,7}$ In this review, I introduce this alternative pathway, which we designated as the futalosine pathway. ${ }^{7}$

\section{HOW IT STARTED}

I have been studying the enzymes and genes responsible for the biosyntheses of isoprenoids produced by actinomycetes. ${ }^{8}$ Approxi- 


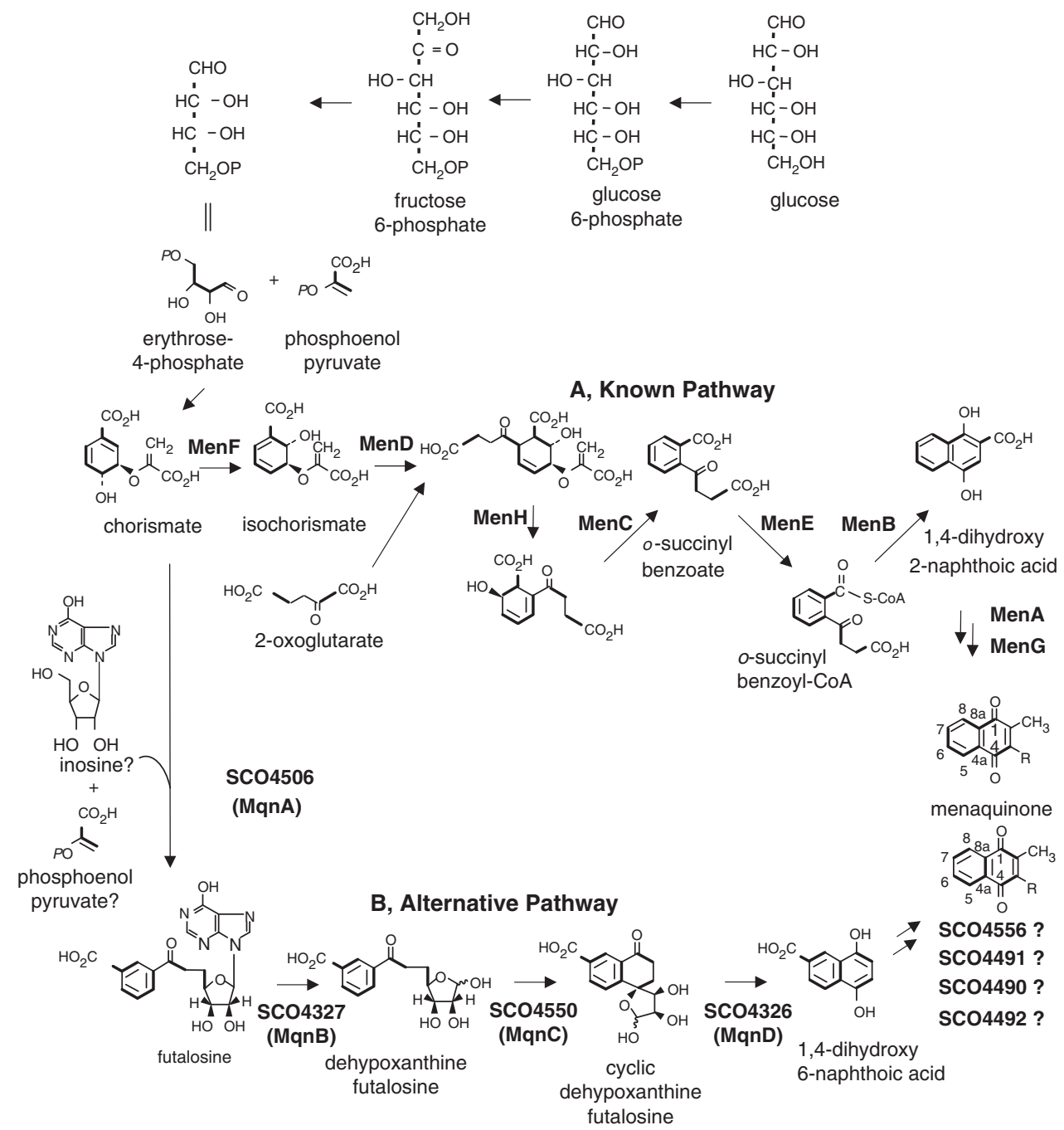

Figure 1 MK biosynthetic pathways. Known pathway (A). Alternative pathway (B). Bold lines in (A) indicate two, three and four carbon units derived from acetate, phosphoenol pyruvate and erythrose-4-phosphate, respectively. Bold lines in (B) show two and four carbon units that were perhaps derived from C-5 and $\mathrm{C}-6$ glucose and $\mathrm{C}-3$ to $\mathrm{C}-6$ glucose (erythrose-4-phosphate), respectively, by the tracer experiment with $\left[\mathrm{U}^{-13} \mathrm{C}_{6}\right]$ glucose.

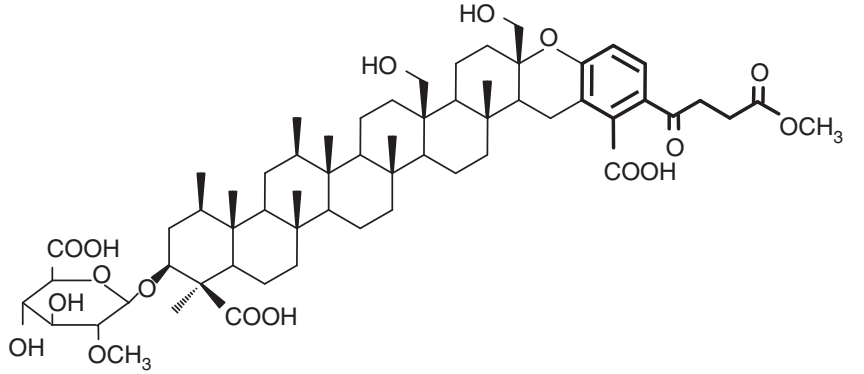

Figure 2 Structure of KS-505a. o-Succinylbenzoate moiety is shown in bold lines.

mately 6 years ago, I tried to clone a gene cluster participating in the biosynthesis of KS-505a (Figure 2), which is produced by Streptomyces argenteolus strain $\mathrm{A}-2$ and has a unique cyclized tetraterpene moiety and an aromatic moiety derived from OSB. ${ }^{9}$ Antibiotic biosynthetic genes cloned from actinomycetes are usually clustered in a genomic DNA region, ${ }^{8}$ and OSB is synthesized by the MenF, -D and -C enzymes in the known MK biosynthetic pathway. ${ }^{1,2}$ Therefore, I first tried to clone men F, -D and -C orthologs from the KS505a producer, as actinomycetes are known to synthesize MK and the length of the prenyl side chain of MK has been used for taxonomic studies of actinomycetes. ${ }^{10}$ To design PCR primes, I searched for the orthologs in databases of Streptomyces coelicolor A3(2) ${ }^{11}$ and Streptomyces avermitilis, $^{12}$ the entire genomes of which have been sequenced. Very curiously, however, I did not detect any orthologs in the two strains. Besides these three orthologs, men $\mathrm{E}$ and $\operatorname{men} \mathrm{B}$ orthologs were also absent in both strains. ${ }^{11,12}$ Some pathogenic microorganisms, such as Helicobacter pylori ${ }^{13}$ and Campylobacter jejuni, ${ }^{14}$ which are known to cause gastric carcinoma and diarrhea, respectively, also lacked these five men gene orthologs, although they synthesize MK. ${ }^{15,16}$ In contrast, these microorganisms possessed orthologs of menA and men $\mathrm{G}^{13,14}$ which catalyze a prenylation and a methylation, respectively, suggesting that the naphthoquinone moiety of MK would be synthesized through an alternative pathway.

\section{TRACER EXPERIMENTS USING [U-13 C]GLUCOSE} AND S. COELICOLOR A3(2)

To investigate whether an alternative pathway was operating in some bacteria, we first analyzed ${ }^{13} \mathrm{C}$-labeled $\mathrm{MK}$ prepared from $S$. coelicolor 
A3(2). ${ }^{6}$ The ${ }^{13} \mathrm{C}-\mathrm{NMR}$ spectrum of MK labeled with $\left[\mathrm{U}-{ }^{13} \mathrm{C}_{6}\right]$ glucose showed a labeling pattern that could be explained by the incorporation of $\left[\mathrm{U}_{-}{ }^{13} \mathrm{C}_{6}\right]$ glucose into $\mathrm{MK}$ through the shikimate pathway by the condensation of phosphoenolpyruvate (PEP) and erythrose4-phosphate (E4P). These two compounds used for A-ring formation were the same as those in the known pathway, but the incorporated positions (PEP into C-7 and -8 , and $\mathrm{E} 4 \mathrm{P}$ into $\mathrm{C}-8 \mathrm{a},-4 \mathrm{a},-5$ and -6 ) differed from those in the known pathway (Figure 1). Therefore, we carried out further experiments using glucose labeled with ${ }^{13} \mathrm{C}$ at different positions. The specific incorporation of $\left[3-{ }^{13} \mathrm{C}\right]-,\left[4-{ }^{13} \mathrm{C}\right]-$, $\left[5-{ }^{13} \mathrm{C}\right]-$ and $\left[6-{ }^{13} \mathrm{C}\right]$ glucose into $\mathrm{C}-8 \mathrm{a},-4 \mathrm{a},-5$ and -6 , respectively, was observed. This labeling pattern is compatible with the above-mentioned findings. The enrichment of C-7 and C-8 by $\left[5-{ }^{13} \mathrm{C}\right]$ glucose and $\left[6-{ }^{13} \mathrm{C}\right]$ glucose, respectively, supported the incorporation of these precursors by PEP through the shikimate pathway. These results established the formation mechanism for the A ring of $\mathrm{MK}$ from the shikimate pathway.

In contrast to the known pathway, no specific incorporation of $\left[1,2-{ }^{13} \mathrm{C}_{2}\right]$ acetate into the $\mathrm{B}$ ring of $\mathrm{MK}$ was observed. However, the precursors of the $\mathrm{B}$ ring could perhaps be PEP (into C-3/C-4) and a currently unknown metabolite originating from C-5 and C-6 of glucose (into C-1/C-2). Taking these results together, we concluded that an alternative pathway was operating, at least $S$. coelicolor $\mathrm{A} 3(2)$.

\section{IN SILICO SCREENING FOR CANDIDATE GENES RESPONSIBLE FOR THE ALTERNATIVE PATHWAY}

We searched for genes responsible for the alternative pathway using two strategies. One was in silico screening, which utilized genome databases, and the other was screening of mutants, which required MK for their growth. First, we searched for candidate genes by comparing two groups of genome sequences. To date, sequencing of the entire genomes of more than 800 microorganisms has been completed. ${ }^{17}$ Among them, we found that some microorganisms belonging to the epsilon and delta categories in Proteobacteria, such as $H$. pylori, Wolinella succinogenes, C. jejuni and Geobacter sulfurreducens, a part of actinobacteria, such as S. coelicolor and S. avermitilis, and certain Deinococcus-Thermus bacteria, such as Thermus thermophilus and Deinococcus radioduran and so on, had no men gene orthologs despite the fact that these strains synthesize MKs. Among them, we selected four microorganisms, H. pylori, ${ }^{13}$ C. jejuni, ${ }^{14}$ T. thermophilus ${ }^{18}$ and $S$. coelicolor, ${ }^{11}$ as query microorganisms. With regard to subject microorganisms, in which the known MK biosynthetic pathway was operating, E. coli, ${ }^{19}$ B. subtilis, ${ }^{20}$ Corynebacterium glutamicum $^{21}$ and Mycobacterium tuberculosis ${ }^{22}$ were selected. First, we searched for orthologs in each of the groups by reciprocal best-hit pairs using the blastp program ${ }^{23}$ with a cutoff value of less than $10^{-10}$. Next, we searched for candidate genes present in the former group but absent in the latter group. We selected approximately 50 genes in $S$. coelicolor A3(2). Among them, putative transcriptional regulator genes and putative membrane proteins for the transportation of small molecules were excluded and four genes, SCO4326, SCO4327, SCO4506 and SCO4550, were selected as final candidates, all of which were reported to be encoded as hypothetical proteins.

\section{GENE DISRUPTION OF THE CANDIDATE GENES}

To examine whether the candidate genes indeed participated in the alternative pathway, we disrupted these genes by homologous recombination. We are experienced at handling Streptomyces strains ${ }^{24}$ and therefore used S. coelicolor A3(2) as a model microorganism. Each of the candidate genes was replaced with a thiostrepton resistance gene by a double-crossover homologous recombination. We were only able to knock out all of the candidate genes when disruptants were selected on agar plates containing MK4 (prenyl side-chain length of C20), which is commercially available and can be substituted for MK8 (prenyl side-chain length of C40) usually found in S. coelicolor A3(2). ${ }^{25}$ The disruption of each of the mutants was confirmed by PCR using specific primers. The SCO4326, SCO4327, SCO4506 and SCO4550 disruptants obligatorily required MK4 for their growth and the MKs purified from these disruptants were confirmed to be MK4 rather than MK8 by HPLC analysis. Considering that SCO4506 and SCO4326 can be mono-cistronically transcribed and exist in the most downstream region of an operon, ${ }^{11}$ the polar effects of the disruptions of these genes would be negligible. Hence, SCO4506 and SCO4326 are essential. In contrast, SCO4550 and SCO4327 were judged to be possibly co-transcribed with SCO4551 and SCO4326 in that order, ${ }^{11}$ respectively, suggesting that the phenotypes of the SCO4550 and SCO4327 disruptants may reflect polar effects. To examine this possibility, plasmids carrying SCO4551 and SCO4326 were introduced into the SCO4550 and SCO4327 disruptants, respectively, and the cell growth rates of the transformants were examined with or without MK4. Eventually, the transformants again required MK4 for their growth, thereby showing that SCO4550 and SCO4327 are also indispensable for the biosynthesis of MK. In summary, we identified four genes (SCO4326, SCO4327, SCO4506 and SCO4550) as alternative MK biosynthetic pathway genes by in silico screenings and genedisruption experiments.

\section{ISOLATION OF MUTANTS BY MUTAGENESIS}

We could not narrow down any additional candidate genes by bioinformatics and therefore tried to isolate mutants that required MK4 for their growth by $N$-methyl- $N^{\prime}$-nitro- $N$-nitrosoguanidine mutagenesis to obtain all the genes participating in the alternative pathway. We were able to isolate 26 mutants, and most of these were complemented by plasmids carrying the genes identified by the in silico screening. A few mutants that were not complemented were used as hosts for shotgun cloning experiments, and all of these were complemented by a plasmid carrying the SCO1490-SCO1501 genes. By subcloning experiments, these mutants were again complemented by the SCO1494, SCO1495 and SCO1496 genes, which encode 3-dehydroquinate synthase, shikimate kinase I and chorismate synthase, respectively (Figure 3 ). The mutant was then spread on a plate containing shikimate and chorismate instead of MK4, and its

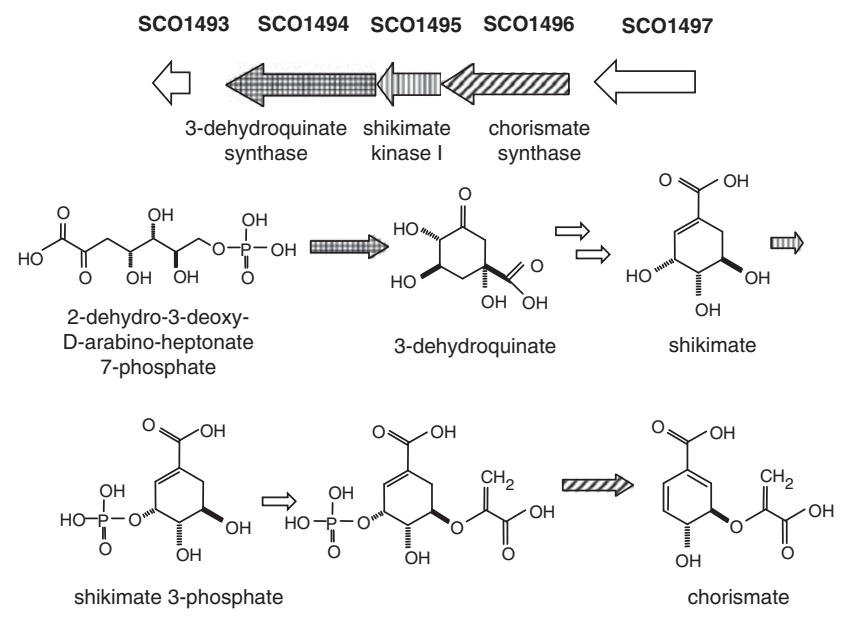

Figure 3 SCO genes that complemented MK auxotroph. Organization of the genes and their functions in the shikimate pathway are shown. 

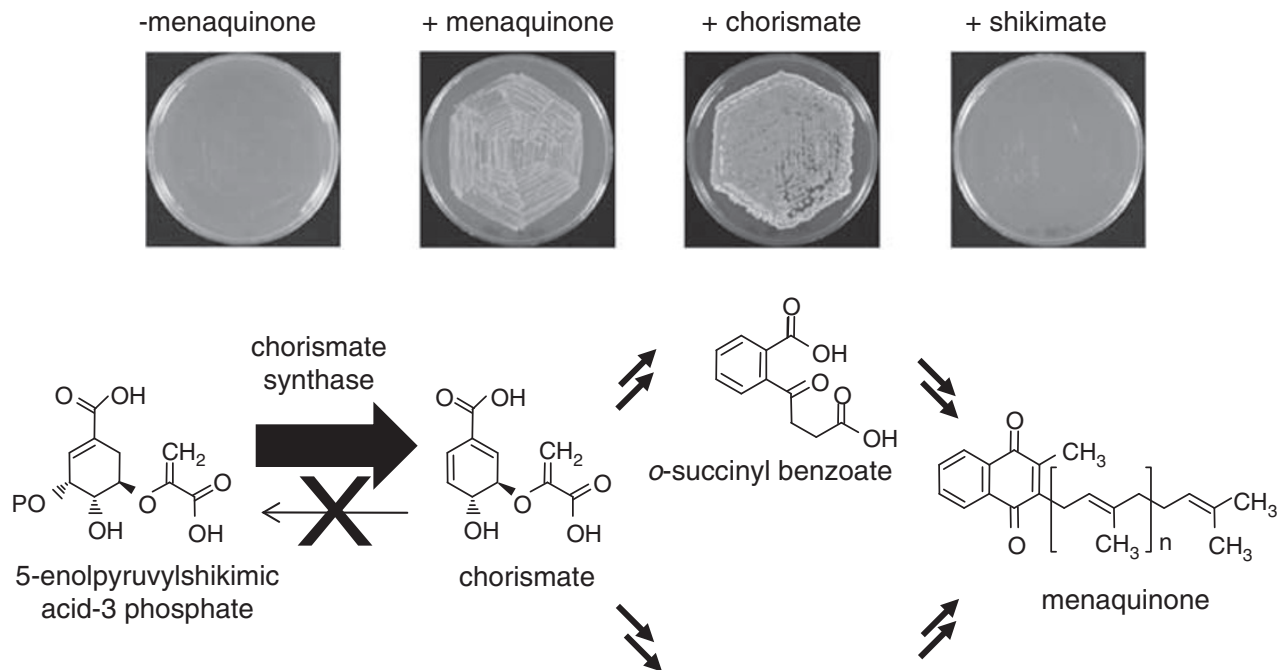

Sc04506, Sc04326, Sc04327, Sc04550

Figure 4 Phenotypes of MK auxotroph. The mutant used for shotgun cloning experiment was spread onto a yeast extract-malt extract (YEME) plate, and a YEME plate containing MK4, chorismate or shikimate. After cultivation at $30^{\circ} \mathrm{C}$ for 1 week, photographic images of each plate were taken. Irreversible reaction catalyzed by chorismate synthase is schematically shown.

growth was examined. The mutant only grew in the presence of chorismate (Figure 4), suggesting that it lacked a functional chorismate synthase or shikimate kinase. As the biosynthetic reaction catalyzed by chorismate synthase is irreversible, ${ }^{26}$ the alternative pathway appeared to be branched at chorismate in a similar manner to the known pathway, but then becomes diverged.

\section{RELATIVE BLOCKED POINTS OF THE DISRUPTANTS}

As described above, we constructed SCO4326, SCO4327, SCO4506 and SCO4550 disruptants. We investigated whether intermediates accumulated in the culture broth of these disruptants. We cultivated each of the mutants in the presence of MK4. The culture broth was centrifuged and MK4 was removed by ethyl acetate extraction. The aqueous layer concentrated by freeze-drying was added into agar plates and the growth of the other mutants was examined. When plates containing the extract from the SCO4506 disruptant were used, the other disruptants showed no growth, suggesting that the SCO4506 product may participate in the most upstream step in the pathway. In contrast, at least one disruptant became able to grow on plates containing the extracts from the other disruptants. On the basis of these results, the relative blocked points of the mutants in MK biosynthesis were estimated to be in the following order: SCO4506, SCO4327, SCO4550 and SCO4326.

INTERMEDIATE COMPOUNDS IN THE ALTERNATIVE PATHWAY Isolation of the accumulated metabolite in the culture broth of the SCO4327 disruptant

Next, we purified the accumulated intermediate in the culture broth of the SCO4327 disruptant using the assay method described above. The intermediate was purified from the MK4-free aqueous layer of the culture broth of the SCO4327 disruptant by Dowex ion-exchange resin column chromatography (Dow Chemical Company, Midland, MI, USA) and a successive DIAION HP20 resin column chromatography (Mitsubishi Chemical Corporation, Tokyo, Japan). Finally, the compound was fractionated by preparative HPLC. By NMR and mass spectra analyses, the compound was confirmed to be futalosine, which had previously been isolated from the culture broth of a Streptomyces strain. ${ }^{27}$

\section{Conversion of futalosine by a recombinant enzyme}

As futalosine was isolated from the culture broth of the SCO4327 disruptant, the SCO4327 product should catalyze the conversion of futalosine into the next intermediate. Hence, we performed in vitro assays using a recombinant enzyme. We first prepared SCO4327 as a recombinant $N$-terminal His-tagged protein and subjected it to an in vitro assay with futalosine as a substrate. However, no products were formed despite the addition of various cofactors and metals to the reaction mixture. We do not know the exact reason why the recombinant protein showed no enzymatic activity, but we assume that it may have been unstable. Therefore, we prepared a recombinant TTHA0556, which is an ortholog of SCO4327 in T. thermophilus $\mathrm{HB} 8{ }^{18}$ an extreme thermophile, expecting that the recombinant protein would be highly thermostable. When purified $\mathrm{N}$-terminal maltose-binding protein (MBP)-fused TTHA0556 was incubated with futalosine, the substrate was quickly consumed and two reaction products emerged in the absence of cofactors and metals. As SCO4327 and TTHA0556 products have very weak similarities to some nucleosidases, we expected that the TTHA0556 product may release hypoxanthine from futalosine. Indeed, one product was confirmed to be hypoxanthine by liquid chromatography-mass spectrometry (LC-MS) analysis. The other product was purified by reverse-phase preparative HPLC and its structure was determined by mass spectra and NMR spectra analyses to be dehypoxanthine futalosine (DHFL). DHFL was also confirmed to be a real intermediate in the alternative pathway by a bioassay using the SCO4506 disruptant.

Isolation of the accumulated metabolite in the culture broth of the SCO4326 disruptant

As described above, the reaction next to SCO4327 was judged to be possibly catalyzed by SCO4550. Indeed, a small amount of DHFL was accumulated in the culture broth of the SCO4550 disruptant. Hence, we carried out an in vitro enzyme assay with a recombinant enzyme. We prepared both recombinant SCO4550 and recombinant TTHA1092, an ortholog of SCO4550 in T. thermophilus HB8, and incubated them with DHFL. However, we did not detect any products 
Table 1 Distribution of the alternative pathway

\begin{tabular}{|c|c|c|c|c|}
\hline Eubacteria & & Genus & Archaea & Genus \\
\hline \multirow[t]{15}{*}{ Proteobacteria } & $\begin{array}{l}\text { Epsilon } \\
\text { category }\end{array}$ & Helicobacter & Euryarchaeota & Archaeoglobus \\
\hline & & Wolinella & & Thermoplasma \\
\hline & & Thiomicrospira & & \\
\hline & & Campylobacter & Crenarchaeota & Ignicoccus \\
\hline & & Arcobacter & & Pyrobaculum \\
\hline & & Nitratiruptor & & Caldivirga \\
\hline & & Sulfurovum & & Thermoproteus \\
\hline & & Nautilia & & Nitrosopumilus \\
\hline & $\begin{array}{l}\text { Delta } \\
\text { category }\end{array}$ & Geobacter & & \\
\hline & & Pelobacter & & \\
\hline & & Desulfovibrio & & \\
\hline & & Desulfococcus & & \\
\hline & & Desulfobacterium & & \\
\hline & & Anaeromyxobacter & & \\
\hline & & Syntrophobacter & & \\
\hline \multirow{2}{*}{ Acidobacteria } & Acidobacteria & Acidobacteria & & \\
\hline & & Solibacter & & \\
\hline \multirow[t]{10}{*}{ Firmicutes } & Bacillales & Bacillus halodurans & & \\
\hline & & Bacillus clausii & & \\
\hline & Clostridia & Symbiobacterium & & \\
\hline & & Syntrophomonas & & \\
\hline & & Carboxydothermus & & \\
\hline & & Desulfotomaculum & & \\
\hline & & Pelotomaculum & & \\
\hline & & Desulforudis & & \\
\hline & & Heliobacterium & & \\
\hline & & Moorella & & \\
\hline \multirow[t]{4}{*}{ Actinobacteria } & & Streptomyces & & \\
\hline & & Frankia & & \\
\hline & & Acidothermus & & \\
\hline & & Salinispora & & \\
\hline Planctomyces & & Rhodopirellula & & \\
\hline \multirow[t]{2}{*}{ Chlamydia } & & Chlamydia & & \\
\hline & & Chlamydophila & & \\
\hline Spirochete & & Leptospira & & \\
\hline $\begin{array}{l}\text { Green nonsulfur } \\
\text { bacteria }\end{array}$ & & Herpetosiphon & & \\
\hline Deinococcus & & Deinococcus & & \\
\hline \multicolumn{5}{|l|}{ Thermus } \\
\hline & & Thermus & & \\
\hline \multirow{4}{*}{$\begin{array}{l}\text { Hyperthermophilic } \\
\text { bacteria }\end{array}$} & & Aquifex & & \\
\hline & & Hydrogenobaculum & & \\
\hline & & Sulfurihydrogenibium & & \\
\hline & & Thermodesulfovibrio & & \\
\hline
\end{tabular}

with both proteins, even under the various conditions examined. Therefore, we decided to isolate an intermediate from the culture broth of the SCO4326 disruptant, because the reaction product generated by TTHA1092 (SCO4550) would be the same as the intermediate accumulated in the culture broth of the SCO4326 disruptant. The intermediate was purified by almost the same methods as those used for the SCO4327 disruptant. Finally, we isolated one compound and its structure was determined to be cyclic DHFL, as shown in Figure 1.

Conversion of cyclic DHFL by a recombinant enzyme

Next, we examined whether recombinant SCO4326 would convert cyclic DHFL into the next intermediate. In this experiment, we again used a recombinant enzyme of T. thermophilus HB8, expecting that the recombinant protein would be more stable than SCO4326. Recombinant TTHA1568, an ortholog of SCO4326 in T. thermophilus HB8, was prepared as an $N$-terminal MBP-fused protein and was used for an in vitro assay with cyclic DHFL as a substrate. We clearly detected a specific product by HPLC analysis and its structure was confirmed to be 1,4-dihydroxy 6-naphthoic acid (DHNA) by LC-MS analysis.

\section{BIOSYNTHESIS IN THE EARLY STEPS}

As described above, the alternative pathway appeared to branch at chorismate, and the SCO4506 enzyme would participate in the most upstream step. On the basis of the structure of futalosine, the nucleoside moiety would be derived from inosine derivatives. Furthermore, a C2 unit, such as pyruvate or PEP, was previously implicated as a building block between chorismate and inosine $\left(\mathrm{C}^{\prime}\right.$ and $\mathrm{C7}^{\prime}$ positions of futalosine) by tracer experiments. ${ }^{6}$ Therefore, we examined whether recombinant TTHA0803, an ortholog of SCO4506 in T. thermophilus HB8, catalyzed the formation of futalosine. We incubated the recombinant enzyme with a combination of the substrates. We did not detect the formation of futalosine, but 3-(1-carboxyvinyloxy)benzoate and m-hydroxybenzoate were formed from chorismate in the absence and presence of flavin mononucleotide, respectively. It was unclear why the recombinant enzyme showed unexpected activities at this stage. As recombinant TTHA0803 accepted chorismate as a substrate, the nucleosides and C2 units used in this assay may not be the actual substrates, or additional enzymes may be necessary to complete the reaction. Further studies are necessary to clarify this unique biosynthetic step.

\section{BIOSYNTHESIS IN THE LATE STEPS}

Although we did not investigate the biosynthetic steps from DHNA to MK, a putative route can be estimated as follows: SCO4491 and SCO4556 are annotated as the UbiA homolog (prenylation enzyme in ubiquinone biosynthesis and its function would be the same as that of MenA) and MenG homolog (methylation), respectively. ${ }^{11}$ The SCO4491 gene is assumed to constitute an operon with the SCO4490, SCO4492 and SCO4493 genes. Among these, the SCO4493 gene is annotated as an AsnC family transcriptional regulator, which would not directly participate in metabolic reactions. In contrast, SCO4490 and SCO4492 are homologous to UbiX and UbiD, respectively, both of which are known to be decarboxylation enzymes in the ubiquinone biosynthetic pathway, ${ }^{2}$ although $S$. coelicolor A3(2) never synthesizes ubiquinones. ${ }^{10}$ Taking these findings together, the prenylation, methylation and decarboxylation reactions would be catalyzed by SCO4491, SCO4556 and SCO4490/SCO4492, respectively. Moreover, we previously showed that symmetrical compounds, such as 1,4naphthoquinone, were not true intermediates using tracer experiments ${ }^{6}$ and methylation is reported to occur in the final step in the known pathway. Therefore, the order of the later steps could be prenylation, decarboxylation and methylation.

\section{DISTRIBUTION OF THE ALTERNATIVE PATHWAY}

We re-investigated the distribution of the alternative pathway in microorganisms with completely sequenced genomes. ${ }^{17}$ Of the four genes identified in this study (SCO4506, SCO4326, SCO4327 and SCO4550), we identified at least three orthologs in the microorganisms, including Gram-negative/positive microorganisms and Archaea (Table 1). As described above, E. coli has both ubiquinone biosynthetic genes and the known MK biosynthetic genes. However, there were no bacteria possessing both ubiquinone biosynthetic genes and the alternative MK biosynthetic genes. We were also unable 
to find any bacteria equipped with both the known and alternative pathways.

\section{ENZYMATIC PROPERTIES OF FUTALOSINE HYDROLASE}

In addition to $H$. pylori and $C$. jejuni, the following pathogenic bacteria also possess the alternative pathway: ${ }^{17}$ Chlamydia, such as Chlamydophila abortus, Chlamydophila caviae, Chlamydophila felis, Chlamydia muridarum and Chlamydia trachomatis, which cause eye and lung infections and genital diseases; and spirochetes, such as Leptospira borgpetersenii and Leptospira interrogans, which are the most common causes of bovine leptospirosis and also cause zoonotic infections in humans. As humans and commensal intestinal bacteria, including lactobacilli, lack the alternative pathway, a compound that inhibits the alternative pathway would specifically prevent the growth of pathogenic Helicobacter strains without affecting intestinal bacterial flora. Moreover, a rapid and easy diagnosis of $H$. pylori infection that is represented by the urea breath test has been developed. Therefore, a timely medical care for an individual patient with antibiotics specific to the alternative pathway might become possible.

As a first step toward developing such antibiotics, we investigated the enzymatic properties of compounds participating in the futalosine pathway; recombinant TTHA0556 of T. thermophilus, which participates in the second step of the pathway and catalyzes the reaction releasing hypoxanthine from futalosine (we named the enzyme futalosine hydrolase and EC number EC3.2.2.26 was recently assigned to the enzyme), was prepared and used in functional analyses. ${ }^{28}$ Recombinant TTHA0556 formed a homotetramer and only reacted with futalosine. Other structurally related nucleotides and nucleosides were not accepted. Recombinant TTHA0556 required no cofactors or metals, and the optimum $\mathrm{pH}$ and temperature were 4.5 (almost the same activity was observed up to $\mathrm{pH} 6.0$ ) and $80^{\circ} \mathrm{C}$, respectively. The $K_{\mathrm{m}}$ value was calculated to be $154.0 \pm 5.3 \mu \mathrm{M}$ and the $k_{\text {cat }}$ value was $1.02 \mathrm{~s}^{-1}$. Although recombinant TTHA0556 was not inhibited by DHFL, cyclic DHFL or DHNA, it was slightly inhibited by hypoxanthine, with a $K_{\mathrm{i}}$ value of $1.1 \mathrm{~mm}$. As described above, futalosine hydrolase only reacted with futalosine, and was essential for survival. Taking these results together, compounds such as derivatives of hypoxanthine that inhibit enzyme activity can be developed as antiH. pylori drugs.

\section{ACKNOWLEDGEMENTS}

This study was performed in my laboratory, together with Dr T Hiratsuka in collaboration with Professor H Seto (Tokyo University of Agriculture; tracer experiments), Dr K Furihata (The University of Tokyo; structural analyses of intermediates) and Dr J Ishikawa (National Institute of Infectious Diseases; bioinformatic analyses). This work was supported, in part, by Grants-in-Aid for Scientific Research on Priority Areas 'Applied Genomics' from the Ministry of Education, Culture, Sports, Science, and Technology of Japan, the Urakami Foundation, the Skylark Food Science Institute and the Asahi Glass Foundation to TD.
1 Bentley, R. \& Maganathan, R. Biosynthesis of vitamin K (menaquinone) in bacteria. Microbiol. Rev. 46, 241-280 (1982).

2 Meganathan, R. Biosynthesis of menaquinone (vitamin K2) and ubiquinone (coenzyme Q): a perspective on enzymatic mechanisms. Vitam. Horm. 61, 173-218 (2001).

3 Dahlbäck, B. \& Villoutreix, B. O. Regulation of blood coagulation by the protein c anticoagulant pathway: novel insights into structure-function relationships and molecular recognition. Artern. Thromb. Vasc. Biol. 25, 1311-1320 (2005).

4 Adams, J. \& Pepping, J. Vitamin K in the treatment and prevention of osteoporosis and arterial calcification. Am. J. Health Syst. Pharm. 62, 1574-1581 (2005).

5 Lamson, D. W. \& Plaza, S. M. The anticancer effects of vitamin K. Altern. Med. Rev. 8, 303-318 (2003)

6 Seto, H. et al. Studies on a new biosynthetic pathway for menaquinone. J. Am. Chem. Soc. 130, 5614-5615 (2008).

7 Hiratsuka, T. et al. An alternative menaquinone biosynthetic pathway operating in microorganisms. Science 321, 1670-1673 (2008).

8 Dairi, T. Studies on biosynthetic genes and enzymes of isoprenoids produced by actinomycetes. J. Antibiot. 58, 227-243 (2005).

9 Nakanishi, S. et al. KS-505a, a novel inhibitor of bovine brain $\mathrm{Ca} 2+$ and calmodulindependent cyclic-nucleotide phosphodiesterase from Streptomyces argenteolus. J. Antibiot. 45, 341-347 (1992).

10 Embley, T. M. \& Stackebrandt, E. The molecular phylogeny and systematics of the actinomycetes. Annu. Rev. Microbiol. 48, 257-289 (1994).

11 Bentley, S. D. et al. Complete genome sequence of the model actinomycete Streptomyces coelicolor A3(2). Nature 417, 141-147 (2002).

12 Omura, S. et al. Genome sequence of an industrial microorganism Streptomyces avermitilis: deducing the ability of producing secondary metabolites. Proc. Natl. Acad. Sci. USA 98, 12215-12220 (2001).

13 Tomb, J. F. et al. The complete genome sequence of the gastric pathogen Helicobacter pylori. Nature 388, 539-547 (1997).

14 Parkhill, J. et al. The genome sequence of the food-borne pathogen Campylobacter jejuni reveals hypervariable sequences. Nature 403, 665-666 (2000).

15 Marcelli, S. W. et al. The respiratory chain of Helicobacter pylori: identification of cytochromes and the effects of oxygen on cytochrome and menaquinone levels. FEMS Microbiol. Lett. 138, 59-64 (1996).

16 Moss, C. W., Lambert-Fair, M. A., Nicholson, M. A. \& Guerrant, G. O. Isoprenoid quinones of Campylobacter cryaerophila, C. cinaedi, C. fennelliae, C. hyointestinalis, $C$. pylori, and C. upsaliensis. J. Clin. Microbiol. 28, 395-397 (1990).

$17 \mathrm{http}: / /$ www.genome.ad.jp/kegg/catalog/org_list.html.

18 Henne, A. et al. The genome sequence of the extreme thermophile Thermus thermophilus. Nat. Biotechnol. 22, 547-553 (2004).

19 Blattner, F. R. et al. The complete genome sequence of Escherichia coli K-12. Science 277, 1453-1474 (1997).

20 Kunst, F. et al. The complete genome sequence of the Gram-positive bacterium Bacillus subtilis. Nature 390, 249-256 (1997).

21 Ikeda, M. \& Nakagawa, S. The Corynebacterium glutamicum genome: features and impacts on biotechnological processes. Appl. Microbiol. Biotechnol. 62, 99-109 (2003).

22 Cole, S. T. et al. Deciphering the biology of Mycobacterium tuberculosis from the complete genome sequence. Nature 393, 537-544 (1998).

23 Altschul, S. F., Gish, W., Miller, W., Myers, E. W. \& Lipman, D. J. Basic local alignment search tool. J. Mol. Biol. 215, 403-410 (1990).

24 Hopwood, D. A. et al. Gene Manipulation of Streptomyces, A Laboratory Manual (The John Innes Foundation, Norwich, UK, 1985).

25 Collins, M. D., Pirouz, T., Goodfellow, M. \& Minnikin, D. E. Distribution of menaquinones in actinomycetes and corynebacteria. J. Gen. Microbiol. 100, 221-230 (1977).

26 Macheroux, P., Schmid, J., Amrhein, N. \& Schaller, A. A unique reaction in a common pathway: mechanism and function of chorismate synthase in the shikimate pathway. Planta 207, 325-334 (1999).

27 Hosokawa, N. et al. Futalosine and its derivatives, new nucleoside analogs. Chem. Pharm. Bull. (Tokyo) 47, 1032-1034 (1999).

28 Hiratsuka, T., Itoh, N., Seto, H. \& Dairi, T. Enzymatic properties of futalosine hydrolase, an enzyme essential to a newly identified menaquinone biosynthetic pathway. Biosci. Biotechnol. Biochem. 73, 1137-1141 (2009). 